\title{
Pharmacokinetic Interaction Study of Danshen and Rosuvastatin in Rats and Volunteers
}

\author{
Jie Yang ${ }^{1}$, Junichi Hasegawa' ${ }^{1}$ Yusuke Endo ${ }^{2}$, Kazuhiko litsuka', Miwa Yamamoto ${ }^{3}$, Akiko Matsuda ${ }^{4}$ \\ ${ }^{I}$ Division of Pharmacotherapeutics, Tottori University Faculty of Medical, Japan, ${ }^{2}$ New Medical Research Promotion \\ Center, Tottori University Faculty of Medical, Japan, ${ }^{3}$ Department of Adult and Elderly Nursing, Tottori University \\ Faculty of Medical, Japan, ${ }^{4}$ Faculty of Nursing, Nara Medical University, Japan
}

Background Danshen is a kind of herbal medicine made of the root of salvia miltiorrhiza. It is used prevalently for treatment of cardiovascular diseases and microcirculation disturbance. In the therapy of hyperlipidemia, statins often combine with danshen containing drug so as to have a better prognosis. The study of interaction between statins and danshen is useful for drug combination.

Methods In animal experiment, a type of in situ intestinal absorption rat model was established. In acute experiment and chronic experiment, rats were treated with rosuvastatin $(0.83 \mathrm{mg} / \mathrm{kg})$, or danshen containing drugs $(0.367 \mathrm{~g} / \mathrm{kg}$ equivalent to crude medicine) combined with rosuvastatin respectively. The blood from the portal vein was collected at 15, 30, 45 min and the blood from heart was collected at 45 min after intestinal injection.

In clinic trial, it was a randomized open label, two-period crossover study in six healthy male volunteers. Volunteers had administration of rosuvastatin with placebo or rosuvastatin with danshen containing drug randomly. The blood samples were collected before drug administration and at $0.5,1,1.5,2,4,8,12$ hours after administration.

The concentration of rosuvastatin was measured by LC/MS/MS analysis.

Results In animal experiment, compared with the rosuvastatin only, danshen extract reduced the concentration by 84.4, $84,64.4,50 \%$ at $15,30,45 \mathrm{~min}$ and heart at $45 \mathrm{~min}$. Danshen formulation had a similar effect to and danshen extraction. In the chronic experiment, danshen significantly reduced the absorption of rosuvastatin, however, between the groups pretreated with danshen (group A and B), or the groups injected with rosuvastatin combined danshen (group B and D), the absorption of rosuvastatin was not changed.

In clinic trial, with co-administration of Danshen, the maximum plasma concentration ( $\mathrm{Cmax}$ ) was decreased by $26.85 \%$ compared with rosuvastatin only and the area under the plasma concentration-time curve from 0 to $12 \mathrm{~h}$ (AUC0-12) was decreased by $12.12,40.22,28.98,15.64,21.80,17.81 \%$ respectively.

Conclusion The results of animal experiment and clinic trail revealed that danshen could reduce the absorption of rosuvastatin in rats and volunteers significantly. It is better to avoid combination of Danshen containing drugs with rosuvastatin in therapy of cardiovascular diseases. 